\title{
AVALIAÇÃO DA EFICÁCIA DE UM PROGRAMA DE AULAS DE EDUCAÇÃO FÍSICA NO DESENVOLVIMENTO COGNITIVO DE CRIANÇAS ABRIGADAS
}

\author{
Rita de Cássia de Souza de Araújo, Universidade Castelo Branco - UCB, Rio de Janeiro, Rio de \\ Janeiro - Brasil \\ Igor da Silva Carvalho, Universidade Castelo Branco - UCB, Rio de Janeiro, Rio de Janeiro - Brasil \\ Marcos Vinicius Marques do Nascimento, Universidade Castelo Branco - UCB, Rio de Janeiro, Rio de \\ Janeiro - Brasil \\ Iris Lima e Silva, Universidade Castelo Branco - UCB, Rio de Janeiro, Rio de Janeiro - Brasil \\ Fabrício Cardoso, Universidade Castelo Branco - UCB, Rio de Janeiro, Rio de Janeiro - Brasil \\ Heron Beresford, Universidade Castelo Branco - UCB, Rio de Janeiro, Rio de Janeiro - Brasil
}

\section{RESUMO}

O presente estudo foi submetido ao Comitê de Ética em Pesquisa envolvendo Seres Humanos da Universidade Castelo Branco - RJ, sendo aprovado sob protocolo ${ }^{\circ}$. 0175/2008 e teve por objetivo avaliar a eficácia de um programa de aulas de educação física, para atuar no atraso do desenvolvimento cognitivo de crianças abrigadas. A amostra foi composta por seis crianças com cinco anos, abrigadas na Sociedade Civil de Amparo ao Menor-Abrigo, no Rio de Janeiro. A metodologia teve formato experimental, operacionalizado por meio de um sistema de avaliações efetuadas antes e depois da intervenção proposta. Como estratégia metodológica para avaliar o nível de organização das estruturas cognitivas das crianças foi utilizada o conjunto de 'Provas Operatórias' propostas por Piaget (1975, apud WEISS, 2001), classificando-se o nível de organização das estruturas cognitivas das crianças. O programa de intervenção constou de 36 aulas de educação física, com enfoque na ludoergomotricidade e duração de 120 minutos cada, desenvolvidas em um período de quatro meses. Incluiu jogos de raciocínio lógico, atenção e memória, atividades para o desenvolvimento de habilidades motoras como andar, correr, pular, deslizar, saltar, rodar, rastejar, subir e, ainda, de desenvolvimento de valências físicas tais como força, velocidade, ritmo e coordenação. A partir dos resultados pôde-se concluir pela eficácia da intervenção educacional aplicada, tendo em vista a maior maturação das estruturas cognitivas observada na segunda avaliação.

Palavras-Chave: Desenvolvimento cognitivo; Crianças abrigadas; Aulas de Educação Física; Ludoergomotricidade. 


\title{
EFFECTIVENESS'S EVALUATION OF A PHYSICAL EDUCATION CLASSES PROGRAM INCOGNITIVE DEVELOPMENT OF CHILDREN SHELTERS
}

\begin{abstract}
The current study was submitted to the Committee of Ethics in Research involving Human Beings in the Castelo Branco University - RJ, being approved under protocol number 0175/2008 and its object was to evaluate the effectiveness of a program of physical education classes, to act on the cognitive development delay of sheltered children. The sample was comprised of six five-year-old children, sheltered on the (shelter for minors) Sociedade Civil de Amparo ao Menor-Abrigo, in Rio de Janeiro. The methodology had an experimental format, operationalized by means of a system of evaluations carried out before and after the proposed intervention. As a methodological strategy to evaluate the level of organization of the children's cognitive structure, the set of "Operational Tests" proposed Piaget (1975, apud WEISS, 2001) was used, rating the level of organization of the children's cognitive structure. The intervention program consisted of 36 physical education classes, focusing on ludoergomotricidade and duration of 120 minutes each, developed over a period of four months. It included games of logical reasoning, attention and memory, activities for developing motor skills like walking, running, jumping, sliding, jumping, running, crawling, climbing, and also development of valences such as physical strength, speed, pace and coordination. From the results, the effectiveness of the applied educational intervention can be favorably concluded, in view of the greater maturation of the cognitive structures observed in the second evaluation.
\end{abstract}

Key-Words: Cognitive development; Sheltered children; Physical Education classes; Ludoergomotricidade. 


\section{INTRODUÇÃO}

No Brasil, de acordo com Rizzini, ${ }^{1}$ o número de crianças em situação de abandono pela família, com conseqüente abrigamento, é alarmante. Este, diante da inexistência de uma rede protetiva adequada, ainda é o meio mais fácil para resolver questões relacionadas a conflitos familiares, desde os mais simples até os mais complexos, como abuso sexual, negligência e maus tratos,

Muito embora seja uma solução, o abrigamento pode comprometer o desenvolvimento normal da criança, gerando riscos de prejuízos físicos, psíquicos e sociais para aquelas. Isto se deve, em parte, à quebra de uma rotina constituída anteriormente e da necessidade de adaptação à nova realidade na qual a criança sofre falta de proximidade com outras pessoas e mesmo insegurança. Geralmente, quando isto ocorre aquela pode mostrar falta de iniciativa, baixa auto-estima, dificuldades de sociabilidade e de aprendizagem, dentre outros problemas. ${ }^{2,3}$

Estudiosos como Fonseca $^{4}$ e Freire $^{5}$ observam que existe, uma correlação entre o desenvolvimento neuromotor e o desenvolvimento em geral de crianças. $\mathrm{O}$ ato motor oferece a possibilidade de deslocamento no tempo e no espaço, oportunizando experiências que incrementam a independência e o sentimento de segurança. Oferta também estrutura necessária para que as emoções se expressem em atitudes corporais, facilitando a comunicação intra e interpessoal ao indivíduo. ${ }^{6}$

A importância de oferecer oportunidade da criança se manter em movimento para que tenha um efetivo desenvolvimento já era preconizada por Platão apud Sheridan. ${ }^{7}$ Portanto, nada Conexões: revista da Faculdade de Educação Física da UNICAMP, Campinas, v. 9, n. 1, p. 24-36, jan./abr. 2011. ISBN: 1983-9030 
melhor do que lhe proporcionar atividades físicas com jogos, que contribuam para o processo de desenvolvimento das estruturas cognitivas, permitindo-lhe compreender melhor o mundo. ${ }^{5,8}$

Acredita-se então, que o desenvolvimento cognitivo da criança se constrói a partir de experiências motoras. As funções motoras (movimento) não podem ser separadas do desenvolvimento intelectual (memória, atenção, raciocínio) nem da afetividade (emoções e sentimentos). Para que o ato de ler e escrever se processem adequadamente é indispensável o domínio de habilidades a eles relacionadas, considerando-se que estas são fundamentais manifestações ludomotoras.

Nesse contexto, brincar é uma necessidade básica da personalidade, do corpo e da mente; faz parte das atividades essenciais da dinâmica humana que se caracterizam por serem espontâneas, funcionais, satisfatórias e, por isto, necessárias à plena formação do organismo de uma criança, de seu intelecto e de sua personalidade. Nas brincadeiras a criança experimenta pessoas e coisas, armazena memória, estuda causas e efeitos, resolve problemas, constrói um vocabulário útil, aprende a controlar as reações emocionais e adapta seus comportamentos aos hábitos culturais do grupo social. ${ }^{8,5}$

Segundo Moyles, ${ }^{9}$ o brincar também possibilita que o cérebro se mantenha ativo. Neste ato o participante sente-se desafiado a dominar o que já lhe é conhecido, como também busca investigar o que de novo a situação lhe propõe. Em função disso, o brincar, em todas as idades, é realizado com prazer e em uma atitude positiva diante da vida.

Conexões: revista da Faculdade de Educação Física da UNICAMP, Campinas, v. 9, n. 1, p. 24-36, jan./abr. 2011. ISBN: 1983-9030 


\section{OBJETIVO}

A partir do exposto, o objetivo deste estudo foi avaliar a eficácia de um programa de aulas de educação física, para atuar no desenvolvimento cognitivo de crianças abrigadas.

\section{ÉTICA DA PESQUISA}

O projeto desta pesquisa foi submetido ao Comitê de Ética em Pesquisa envolvendo Seres Humanos da Universidade Castelo Branco (UCB/RJ) e aprovado sob protocolo nº . 0175/2008.

A coleta de dados se deu conforme a resolução 196/96 do Conselho Nacional de Saúde. Assim, inicialmente foi solicitada autorização dos pais ou responsáveis para que o estudo fosse desenvolvido, através de um Termo de Consentimento Livre e Esclarecido, ao qual se anexou uma carta de esclarecimentos, explicando-se a natureza estudo

\section{MÉTODO}

O presente estudo teve o formato experimental, entendendo-se que uma pesquisa experimental consiste em determinar um objeto de estudo, selecionar as variáveis que seriam capazes de influenciá-lo, definir as formas de controle e de observação dos efeitos que a variável independente produz diretamente na variável dependente, isto sendo realizado em um sistema de avaliações efetuadas antes e depois da intervenção proposta.

Como estratégia metodológica para avaliar o nível de organização das estruturas cognitivas das crianças foi utilizado o conjunto de 'Provas Operatórias' propostas por Piaget apud Weiss, ${ }^{10}$ segundo descrito no Quadro 1, nos momentos antes e após a intervenção.

Conexões: revista da Faculdade de Educação Física da UNICAMP, Campinas, v. 9, n. 1, p. 24-36, jan./abr. 2011. ISBN: 1983-9030 
Quanto ao grupo estudado, este configurou um censo, segundo Cochran, ${ }^{11}$ abrangendo seis crianças com cinco anos abrigadas na Sociedade Civil de Amparo ao Menor-Abrigo a Minha Casa (RJ), sob tutela do Estado e disponíveis para adoção.

Quadro 1 - Padrão de Desenvolvimento de Piaget adaptado (WEISS, 2001).

\begin{tabular}{cccc}
\hline \multicolumn{4}{c}{ Padrão de desenvolvimento esperado } \\
\hline Idade & Conservação & Classificação & Ordenação \\
05 anos & Nível 2 & Nível 2 & Nível 2 \\
\hline
\end{tabular}

Fonte: Adaptado de WEISS, 2001.

A avaliação da capacidade "conservação" foi realizada a partir do "Teste de Pequenos Conjuntos

Para a avaliação da capacidade de classificar os objetos foi aplicada a prova de mudança de critério ou dicotomia, tendo fichas de figuras geométricas de formas e cores diferentes como recurso.

Já a etapa da 'ordenação' foi avaliada por meio da prova de seriação de bastonetes.

A aplicação de todas as provas seguiu o procedimento padrão proposto pelo autor, bem como sua interpretação, classificando-se três tipos de respostas: se obtido êxito na prova - quando a criança apresentava argumentos operatórios - a classificação foi nível 2; se observada uma conduta intermediária - quando às vezes apresentava argumentos operatórios e às vezes não - 
aquela foi estabelecida como nível 1; e se identificada a ausência da capacidade - quando não apresentava argumentos operatórios - foi estabelecida como nível zero.

Foi plicado o teste de Wilcoxon para dados pareados, no sentido de verificar a significância estatística das diferenças entre os resultados do primeiro e do segundo instantes de avaliação, para cada capacidade.

\section{PROCEDIMENTOS DE INTERVENÇÃO}

O programa constou de 36 aulas de educação física, com enfoque na ludoergomotricidade e duração de 120 minutos cada, desenvolvidas em um período de quatro meses. Incluiu jogos de raciocínio lógico, atenção e memória, atividades para o desenvolvimento de habilidades motoras como andar, correr, pular, deslizar, saltar, rodar, rastejar, subir e, ainda, de desenvolvimento de valências físicas tais como ritmo e coordenação.

\section{RESULTADOS}

A Figura 1 mostra o desempenho das crianças nos momentos pré e pós intervenção, quanto à capacidade de 'conservação'. Os resultados da avaliação inicial mostraram que das seis crianças, somente uma apresentou o padrão esperado (2 pontos), revelando pequeno nível de organização quanto a esta capacidade cognitiva. Já na avaliação de produto este resultado foi alcançado por cinco crianças, enquanto uma, a mesma que perfizera dois pontos inicialmente, conseguiu atingir um escore de 3 pontos, ou seja, um resultado acima do esperado.

Conexões: revista da Faculdade de Educação Física da UNICAMP, Campinas, v. 9, n. 1, p. 24-36, jan./abr. 2011. ISBN: 1983-9030 


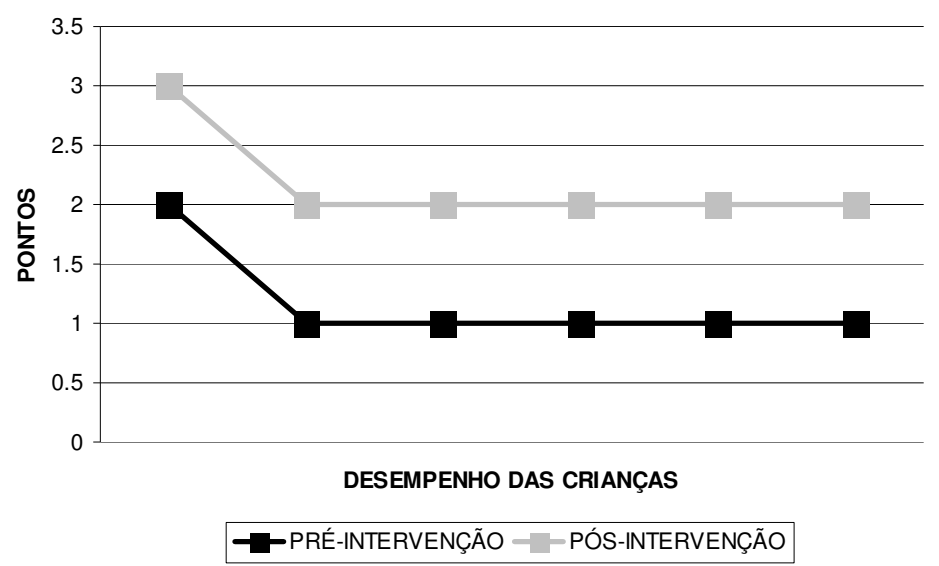

Figura 1- Desempenho das crianças nos momentos pré e pós intervenção, quanto à capacidade de 'conservação'

Quanto à capacidade de classificar objetos, na primeira avaliação três crianças apresentaram um nível operacional de acordo com o estabelecido pelo protocolo, enquanto as outras três apresentaram resultado inferior. A partir da Figura 2 pôde-se observar que após o período de intervenção quatro crianças conseguiram atingir o nível dois, e ainda que duas delas obtivessem um resultado igual a três pontos.

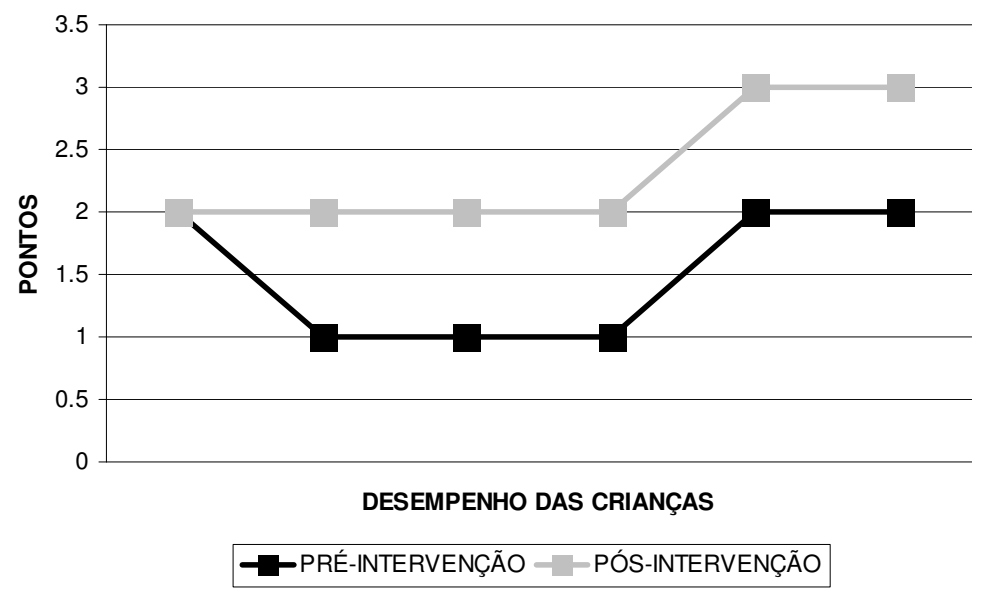

Figura 2- Desempenho das crianças nos momentos pré e pós intervenção, quanto à capacidade de classificar

Conexões: revista da Faculdade de Educação Física da UNICAMP, Campinas, v. 9, n. 1, p. 24-36, jan./abr. 2011. ISBN: 1983-9030 
Para a capacidade operacional de ordenar, inicialmente nenhuma criança apresentou um resultado compatível ao índice padrão estabelecido por Piaget. Porém, após o período de intervenção, tal índice foi alcançado por cinco delas, o que sugere uma evolução.

A significância das diferenças entre o desempenho das crianças, antes e após a intervenção pedagógica, foi confirmada por meio do Teste de Wilcoxon para dados pareados, para todas as capacidades avaliadas, isto é, conservação, classificação e ordenação, encontrando-se valores de $\mathrm{p}=0,02, \mathrm{p}=0,04$ e $\mathrm{p}=0,04$ respectivamente para cada uma delas.

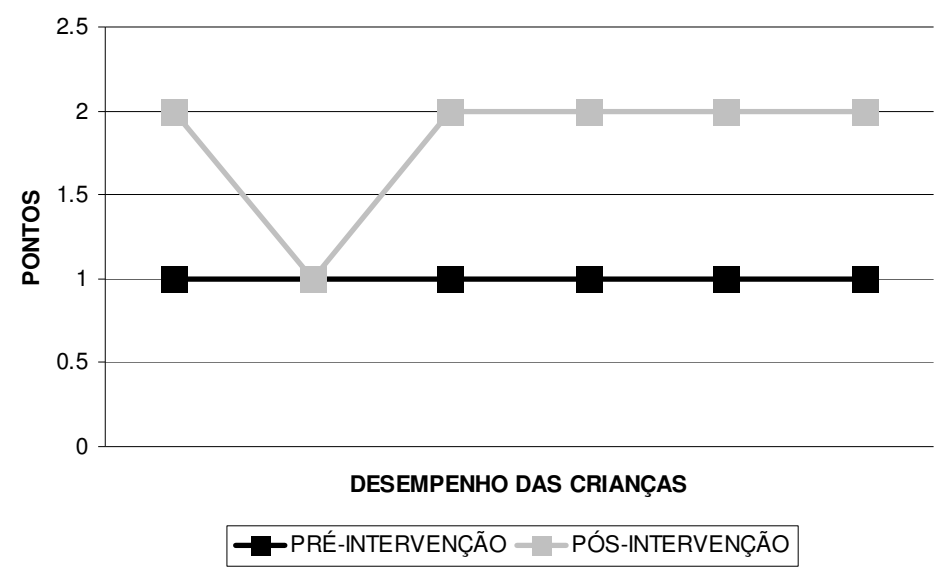

Figura 3- Desempenho das crianças nos momentos pré e pós intervenção, quanto à capacidade de ordenar

\section{DISCUSSÃO}

Os resultados iniciais das provas operatórias aplicadas neste estudo permitiram identificar que havia uma defasagem no funcionamento e o desenvolvimento das funções lógicas das crianças participantes deste estudo, em relação à sua idade cronológica, ou seja, estas crianças apresentam uma defasagem cognitiva, o que está de acordo com o estudo realizado por Suehiro, Rueda e Silva, ${ }^{12}$ que detectaram um déficit no sistema perceptivo de crianças abrigadas.

Conexões: revista da Faculdade de Educação Física da UNICAMP, Campinas, v. 9, n. 1, p. 24-36, jan./abr. 2011. ISBN: 1983-9030 
Já a partir dos resultados finais pode-se perceber que em relação à capacidade de conservar e classificar algumas das crianças apresentaram um escore superior ao esperado. Para Piaget, ${ }^{13}$ as capacidades da criança em pensar e compreender a realidade, podem tanto ser antecipadas como ser retardadas sem, no entanto, modificarem-se a ordem de sucessão em que ocorrem estes estágios. Ou seja, é possível uma criança apresentar o nível de pensamento de uma fase imediatamente superior à esperada. Assim, uma criança de cinco anos, para a qual é esperado pensar no nível pré-operatório, pode mostrar um nível de pensamento próprio do período operatório seguinte, que seria o operatório concreto. Esta afirmação corrobora com o que se constatou nas respostas encontradas nos estudos de Sampaio. ${ }^{14-16}$

\section{CONCLUSÃO}

Concluiu-se que crianças abrigadas podem apresentar atraso no desenvolvimento cognitivo, que, no entanto, poderá ser reduzido, desde que seja oferecido um ambiente com estímulos adequados para isto.

Tais crianças, por terem vivido em um ambiente hostil, são suscetíveis a desajustes morais, violência, abuso sexual, desnutrição, consumo de drogas e toda problemática relacionada à negligência precoce e ao abandono, sem uma figura cuidadora que estabeleça vínculos emocionais firmes, sofrem ausência ou insuficiência de estímulos adequados, necessários ao desenvolvimento cognitivo, em particular, e ao desenvolvimento humano em geral.

Conexões: revista da Faculdade de Educação Física da UNICAMP, Campinas, v. 9, n. 1, p. 24-36, jan./abr. 2011. ISBN: 1983-9030 
Entre essas possibilidades de estímulos, destaca-se a alternativa de intervenção por meio das aulas de educação física com condutas e comportamentos motores intencionalmente focados para suprir, positivamente, o referido estado de carência, privação ou vacuidade de tais crianças, não só na dimensão física, mas também na emocional, moral e social que envolvem a vida existencial das mesmas.

Portanto pode-se considerar que o ser humano é um complexo de emoções e ações e que por meio da estimulação corporal recebidas nas atividades ludomotoras, crianças abrigadas poderão apresentar uma melhora em seu desenvolvimento cognitivo.

\section{REFERÊNCIAS}

${ }^{1}$ RIZZINI, I. A institucionalização de crianças no Brasil: percurso histórico e desafios do presente. Rio de Janeiro: Ed. PUC-Rio; São Paulo: Loyola, 2004.

${ }^{2}$ BOWLBY, J. Separação: em apego e perda. São Paulo, Martins Fontes, 1981. v. 2.

${ }^{3}$ WEBER, L. N. D. Aspectos psicológicos da adoção. Curitiba: Juruá, 1999.

${ }^{4}$ FONSECA, V. da. Da filogênese à ontogênese da motricidade. Porto Alegre: Artes Médicas, 1988.

${ }^{5}$ FREIRE, J. B. Educação de corpo inteiro: teoria e prática da Educação Física. São Paulo: Scipione, 1989.

Conexões: revista da Faculdade de Educação Física da UNICAMP, Campinas, v. 9, n. 1, p. 24-36, jan./abr. 2011. ISBN: 1983-9030 
${ }^{6}$ WALLON, H. Do acto ao pensamento. Lisboa: Moraes, 1978.

${ }^{7}$ SHERIDAN, M. D. Brincadeiras espontâneas na primeira infância. 2. ed. São Paulo: Brasil, 1971

${ }^{8}$ FEIJÓ, O. G. Corpo e movimento: uma psicologia para o esporte. Rio de Janeiro: Shape, 1992.

${ }^{9}$ MOYLES, J. R. Só brincar?: o papel do brincar na educação infantil. São Paulo: Artmed, 2002.

${ }^{10}$ WEISS, M. L. L. Psicopedagogia clínica: uma visão diagnóstica dos problemas de aprendizagem escolar. 8. ed. Rio de Janeiro : DP\&A, 2001.

\section{${ }^{11}$ COCHRAN,}

${ }^{12}$ SUHERIRO, A. C. B.; MARIN RUEDA, F. J.; SILVA, M. A. Desenvolvimento perceptomotor em crianças abrigadas e não abrigadas. Paidéia, v. 17, n. 38, p. 431-442.

${ }^{13}$ PIAGET, J. O nascimento da inteligência na criança. Rio de Janeiro: Zahar, 1975.

${ }^{15}$ CAMPOS, L. G. A. Caracterização do desempenho de crianças com transtorno de défict de atenção e hiperatividade (tdah) em provas operatórias: estudos de caso. Psicopedagogia, São Conexões: revista da Faculdade de Educação Física da UNICAMP, Campinas, v. 9, n. 1, p. 24-36, jan./abr. 2011. ISBN: 1983-9030 
Paulo, v. 24, n. 75, p. 218-228, dez.2009.

${ }^{16}$ BAMPI, M. A. M. O método clínico experimental de Jean Piaget como referência para o conhecimento do pensamento infantil na avaliação psicopedagógica. Florianópolis, 2006. Pedagogia em Foco, Rio de Janeiro, 2006. 